\title{
Context-aware fun and games with Bluetooth
}

\author{
Andy Sloane and Chris Dennett
}

School of Computing and IT, University of Wolverhampton, UK

A.Sloane@wlv.ac.uk, C.Dennett@wlv.ac.uk.

\begin{abstract}
Bluetooth-enabled devices are everywhere and in everyday use by a large number of mobile phone users. Harnessing this communication channel for a context-aware system has proved to be a useful step forward in providing simple games, fun events and targeted advertising based on the awareness of the user's location. As part of a wider project the authors have produced a system that allows users to download information based on Global positioning (GPS) inputs on moving server platforms within public transport. The project has also tied "context" to information to send information to users via the same platform based in a static system for applications such as guided trails and tours in open spaces.
\end{abstract}

\section{Introduction}

The system outlined here has two distinct implementations:

1. A system designed to inhabit a mobile space such as a train, tram or bus

2. A system which delivers information based on a user's location in relation to a static system which delivers information with embedded context.

Using Bluetooth is a cost-effective means of providing a delivery system for these mobile information services. The information that can be provided is from a range of multimedia, interactive gaming and other active content. Tie this to the ability to interact with the outside environment via a GPS input and the information and gaming experience can be made "context-aware".

Based on a system [1] used for e-learning support the Bluetooth server design is outlined below. Using this design enabled the project to deliver information based on location information from a GPS input to the server and to frequently 
update the information on the mobile system when in proximity to the project's wireless network.

The purposes of the project were:

- To provide a means of up-to-date information to travellers or tourists

- To provide games and fun items to users via a commonly available platform (mobile phone)

- To provide more serious information such as health education and newsflashes

- To assist users in their travel arrangements

- To act as a means of advertising in a timely and context-driven scenario

- To provide information from static servers with embedded context for later use.

These two different context-based systems required different design strategies and different engagement with the user's device. The use of short bursts of information to the users in a mobile setting (e.g. on a bus or tram) was made possible by the constant "availability" of the users during the journey. Longer bursts of information in the static system were used as the users could engage with the system while entering the experience e.g. buying tickets.

Both systems have been deployed in live environments, with restricted access devices having to be specified (variability of the features and compatibility of the variety of mobile phone platforms does not allow a complete range to be targeted easily), but the intention is to widen the system to cater for more user devices to enable as wide a participation as possible. Other similar systems in the past [2] have used Bluetooth as a location finding tool - this system relies on GPS to do that and only uses Bluetooth as a delivery mechanism it is, therefore restricted. The mobile system relying on an antenna input which does not have too many problems, but the static system uses the phone's own GPS system to deliver context/location information and this relies on the availability of signal in the area of the delivery zone. (Initial "urban canyon" problems restricted the usability of location-driven application in some areas such as the City Centre).

\section{The delivery system}

The system makes use of inexpensive and widely available Bluetooth Universal Serial Bus (USB) 'dongles', and open source software to provide a communications link between the "system" and "users" that requires nothing else, other than a computer, to create a transmission hub or information server. 


\subsection{Bluetooth Technology in Brief}

Bluetooth is a licence-free communications protocol transmitting in the Industrial, Scientific and Medical band and designed for short range ad-hoc networking. Its operation in this band means that it competes with transmissions of all other licence-free short-range systems such as wireless network access points (Wi-Fi). Originally developed by Ericsson in 1994, the standard is now under the control of the Bluetooth Special Interest Group (SIG) [3]. The technology first came to prominence (and is still most commonly associated) with the provision of wireless headsets for mobile phones, but has since been used in a multitude of applications from wireless computer mice to streaming audio.

As an outline, the Bluetooth v1.2 transmission system uses spread-spectrum techniques to reduce the effect of other technologies working in the same band (Wi-Fi being a prime example) on transmitted packets. Transmitting on one frequency for such a short amount of time means that multiple packets are less likely to encounter distortion and there is therefore a reduced need for error control overhead, although a rate $1 / 3$ repetition code and a rate $2 / 3$ block code are available as well as Automatic Repeat Request (ARQ). To further reduce the likelihood of interference, problem frequencies (for instance, those used by static wireless transmitters) are avoided by the systems adaptive algorithm which takes note of frequencies that regularly cause interference to Bluetooth transmission and avoids them for future packet transmissions. Gaussian Frequency Shift Keying (GFSK), a simple and robust digital modulation technique is used for data transmission in Bluetooth v1.2. To increase data throughput, Bluetooth v2.0 uses GFSK only for header information, favouring the increased rate of Quadrature Phase Shift Keying (QPSK), with a guard and synchronisation word, for the payload. This payload rate is further increased through the use of 8-PSK for the Enhanced Data Rate (EDR) that has recently come to market. The ad-hoc network can take on one of two forms. In the first instance, where less than eight devices are present, a piconet is formed. In this situation, one device becomes the master and the others become slaves. All of these devices can communicate with one another simultaneously. If more devices are present, or come within range, slaves can act as masters in other piconets, thus forming a scatternet.

Alongside the radio transmission standard is a range of profiles that are defined by the SIG. These specifications form the backbone to any Bluetooth software development and include profiles for basic services, such as printing and synchronisation, and more involved services, like WAP over Bluetooth and Object Exchange (OBEX). 


\subsection{BlueShoot}

One of the goals of this project was to provide a freely available executable for other members of the community to use for further development. Therefore, the transmission software, BlueShoot, is built to run on a version of the Linux operating system using Python with a MySQL database to keep track of information services permitted by each device, files to be transmitted and whether they have been successfully received or not.

The software, makes use of a number of freely available libraries, primarily PyBluez [4], a Python wrapper for the official Linux Bluetooth software stack (Bluez). This library is used for device discovery. To facilitate the transmission of information to devices using OBEX, and to more easily discover the services on a device, a high-level wrapper for PyBluez and OpenOBEX, LightBlue [5] was used.

Similar systems exist in other environments such as BlueSender for windows but the use of Open Source Software allowed faster development and more flexible utilisation during the project.

\subsection{Software in Practice}

Whereas SMS transmission technologies require nothing more complicated than a phone number, Bluetooth devices require considerably more information. Fortunately most of this information can be obtained via the device software; it only requires that the device is made discoverable for a short time and that the correct profile is permitted without pairing. Once the device has been detected, the user device discovery can be disabled.

The most basic use of the software is transmitting particular files to chosen devices in real time. Once devices have been discovered, the system can transmit a file to a particular device, selection of devices or all devices.

The original prototype sent JPEG images as shown in Figure 1 but this has now been superseded by a richer set of data types available to phone users.

Further extensions to the project will require more extensive communication between the user's phone and the server - for example the context for interactive communication via the server and other phones for multiplayer games requires more data and context information to be stored and different profiles. 


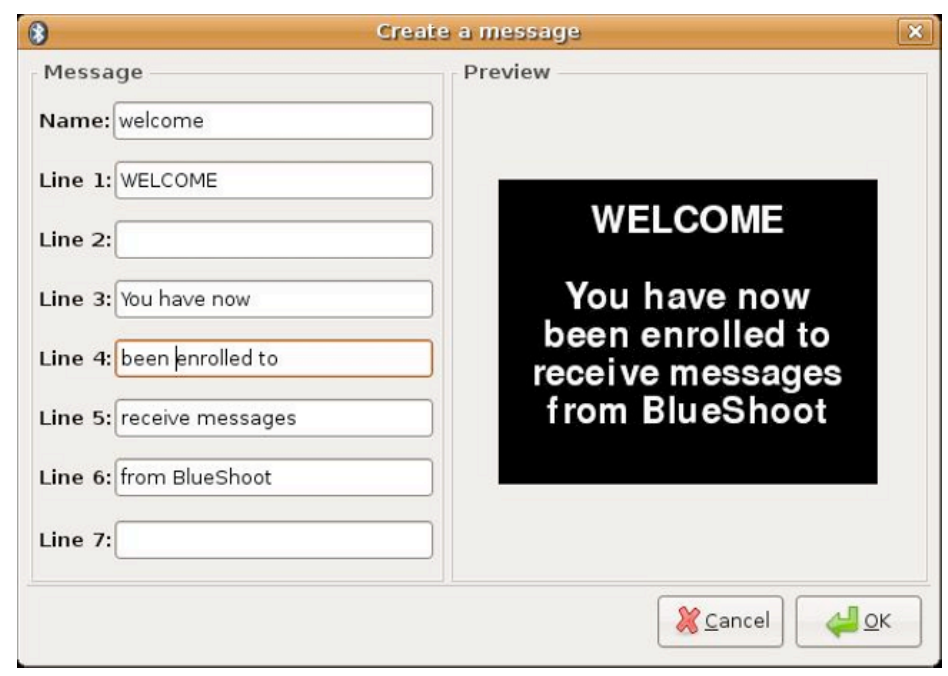

Figure 1. Message screen

\subsection{Server systems}

In the mobile server systems the various data inputs were:

1. Wi-Fi for updating the database on the move and

2. GPS for continuous positioning.

This enables timely data to be transmitted to users and for this to be based on the position of the server geographically. It was therefore possible to devise a system that would deliver information based on the user's location context without them having to have any GPS input on their device. This simplified the user device requirements a little and enabled the project to target a wider range of end-user devices.

The types of service developed for the system ranged from simple animated GIF files, audio files and various combined multimedia data files to active Java applications.

The static server systems were essentially simpler to construct initially as they did not require GPS input, however they did need to have a range of GPS coded trigger points to link to the informational files sent to the user's phones.

The two systems were, therefore, similar in concept but delivery was different with the mobile server system using the GPS input to trigger downloads to users from a wide range of available material; the static system downloaded a limited amount of material with embedded GPS trigger points to a user's phone so that the 
presented information was triggered as the user navigated the geographical space of interest.

A further enhancement was to include user history on an uplink to configure targeted downloads and help reduce the traffic from the server to the user.

\section{User device considerations}

One of the first problems to consider was the range of different user devices (phones and Smart-phones) which are in everyday use. A sample survey was carried out of a student population as this was seen as being fairly representative of active phone users in that they were constant and habitual users of the technology. This survey led to a small number of manufacturers and devices being identified as being useful to target in the first instance with a wider range being left until later in the project for further extension of the system to cover a wider population.

It was also felt that as the project progressed there would be technological developments of the user devices and it was only feasible to target the latest generation of devices as these would soon be widely available at little cost (the UK market being driven partly by free phones available on monthly air-time contracts).

The initial decision on target phone platform was to choose a Nokia N95 8GB - this provided both a modern phone with a large memory capacity and GPS capability within a reasonable size. Some of the Sony Ericsson range were also used in initial tests but the development of software was simplified by the main choice of the S60 $3^{\text {rd }}$ Edition operating system allowing development across a range of target phones.

Further development of the concepts and software will be to expand the range of target platforms to incorporate a wider variety of user devices but choice is left until nearer development time as the rapid obsolescence of mobile devices militates against too much investment in effort at too early a stage.

\subsection{Mobile system}

For the system where the server is mobile the minimum requirements of the user device were Bluetooth and support for animated GIF files. Additional support for Flash-lite and Java enabled richer content to be downloaded and used from the server.

The initial design of the software includes GPS trigger points that then allow information feeds to be downloaded to user phones at relevant points in their journey. This allows relevant advertising and other sponsored content, such as games and quizzes, to be available when the user is in the vicinity of the sponsor. 
The use of a mobile server system allows a large amount of content to be retained and only relevant content delivered at the appropriate location for example as the bus approaches a restaurant it can send a targeted advertisement or a sponsored item to the enabled user phones. This approach allows both opt-in by users and better targeting of material to user requirements. This saves overloading users with too much content material; this may or not be wanted but if it is targeted by location then this allows a coarse filter to be applied. Finer filters can be applied by using an uplink to the server for user input and choice parameters.

Initial applications developed have been animated advertisements and simple games using Flash-lite.

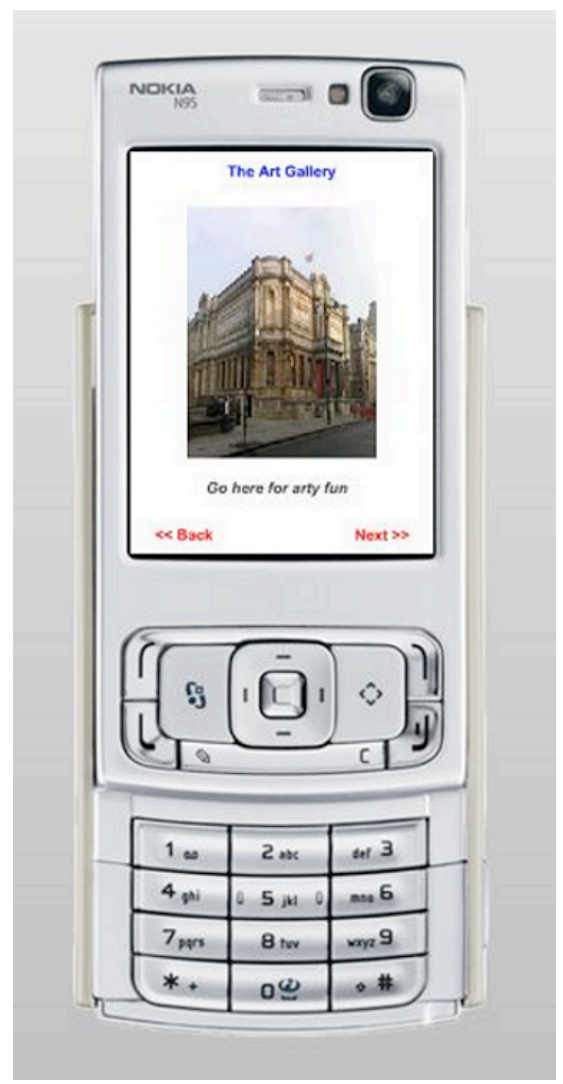

Figure 2. A typical tour screen 


\subsection{Static system}

The additional requirement to make the static system more useful was the inclusion of GPS within the phone. This made the choice of phones a lot more restricted but this was felt to be a temporary problem as many new phones were being predicted with inclusive GPS as an option. It was also felt that Bluetooth/GPSenabled (Personal Digital Assistants) PDAs would be able to be included in the trials as a proof of concept tool but these proved to be too cumbersome to be of more than a passing interest.

The design of this static server system allowed the device to be more compact than the mobile system as generally any of the static systems were only used to deliver a limited number of content streams (mostly a single downloaded file was available at each of the project's "Bluetooth information points").

The initial design was therefore simplified to be a system that downloads a file (with embedded GPS triggers) to the user's device when they interact with the server system. The design was furthered enhanced to included interaction with the user's history of other downloads so that the information could be targeted based on user's context and history data. This is still in development at the time of writing. A typical screen is shown in Figure 2.

\section{Mobile system content}

The moving server utilised the GPS input as a location device. This was found to be accurate enough for the prototype applications. Only some of these relied on $\mathrm{s}$ significant location-awareness. The main locator was the fact that the system, was used in proximity to the users, e.g. on a public tram or bus, and the users were essentially a captive audience for a short time. This allowed the download of a large amount of content to the user's devices as and when it was requested. The use of GPS input to the server allowed a level of location-based sponsorship of the content such as advertising banners on the downloaded games related to the local businesses. This was felt to be a better way to target advertising than to rely on adhoc Bluetooth adverts or similar systems that have been tried in the past. The advantage being that the user can opt-in to the system and is rewarded for participation. 


\section{Static system content}

The significant difference of the static system to the moving system is the reliance on GPS in the user device to provide rich content. This enabled a few different applications to be developed based on the use of tagged GPS multimedia files. This allowed the user to download the complete content (or a large proportion) at a specified point and then take it in the device until the GPS triggered its use in the relevant context.

The specific applications of this technology are quite wide but the concentration at first was the provision of guided trails and annotated multimedia descriptions of displays in open-air museums.

The use of the system will be further described in the final paper and elaborated on in the conference presentation.

One of the applications still under development is a system situated in the foyer of a theatre enabling context-driven advertising to be delivered based on customer preference by linking to the ticketing system - When tickets are sold the customer receives targeted information for similar events based on buying history - Similar to the buying suggestions offered by online retailers.

\section{Analysis of use}

The system has been designed and the prototype will be in full operation as part of a wider context-aware location-based information pilot in. At present (April 2008) only the static content (a guided tour) has been developed as this was seen to be a priority system to develop "proof of concept" and to utilise the capability of the chosen platform. Some initial problems occurred with the delay in obtaining GPS signals and the time required to download the material to the user's device causing limits to be imposed for further development in this type of application. It was felt that the initial download would be improved by being supplemented by further download points in the tour path and so a re-deign was carried out to incorporate the findings. Whilst it is feasible to download the whole tour at the start it was felt that a better interaction with the user could take place if there were a number of different points of contact. This was seen to enhance the design of the software allowing easier integration of future upgrades such as the contextual interaction mentioned above.

A more detailed analysis of the user's reaction to the system will be available at the conference in September as the project is due to end in summer 2008. 


\section{Conclusions}

Initial reactions to the system have been encouraging. Demonstrations have shown a number of possible applications in action that are being further refined and produced as a more polished set of prototype applications. Interest has been shown from a number of different content providers in numerous different areas from simple advertising to more complex interactive e-learning and health education messaging. The number of different applications has had to be curtailed due to limited funds and time to develop but it is envisaged that the prototype will be further developed in the near future to provide a network of useful information points in both moving public areas and static locations in the region, with a variety of enhancements planned to be added for the interaction between the system and the user.

The use of such an information delivery system that allows users to interact via their own phones assists in the promotion of mobile computing systems as there are fewer issues to learning with the user interface as the phone user should be familiar with a number of the available functions already.

A number of further developments are planned as outlined above: These include an "Informational Treasure hunt" where the tour software is enhanced to include an interactive m-learning component based on local context such as historical monuments or buildings.

Another enhancement is to extend the system to include a sub-system to learn the user's context and so provide more closely targeted information to the user's device rather than using a mass download which could include information already consumed at other points or at other times.

Final conclusions on use, usability and final user reactions will be available at the conference in September.

\section{References}

[1] Dennett, C and Traxler J, Project Bluetooth:Support for computing first years, Proc. HEA ICS $8^{\text {th }}$ Annual conference Southampton, UK August 2007, available online at http://www.ics.heacademy.ac.uk/events/8th-annual-conf/

[2] Aalto L, Göthlin N, Korhonen J \& Ojala T Bluetooth and WAP Push based location-aware mobile advertising system. Proc. Second International Conference on Mobile Systems, Applications and Services, Boston, MA, 49 - 58. 2004 Available online at http://www.mediateam.oulu.fi/publications/pdf/496.pdf

[3] Bluetooth Special Interest Group at http://www.bluetooth.com

[4] PyBluez, http://org.csail.mit.edu/pybluez

[5] LightBlue, http://lightblue.sourceforge.net 